\title{
Regression of cephalic index following endoscopic repair of sagittal synostosis
}

\author{
Nicholas A. Pickersgill, BSc, ${ }^{1}$ Gary B. Skolnick, BS, ${ }^{1}$ Sybill D. Naidoo, PhD, RN, CPNP, 1 \\ Matthew D. Smyth, MD, ${ }^{2}$ and Kamlesh B. Patel, MD, MSc ${ }^{1}$ \\ ${ }^{1}$ Division of Plastic and Reconstructive Surgery, Department of Surgery, and 'Department of Neurosurgery, Washington \\ University School of Medicine, St. Louis, Missouri
}

\begin{abstract}
OBJECTIVE Metrics used to quantify preoperative severity and postoperative outcomes for patients with sagittal synostosis include cephalic index (Cl), the well-known standard, and the recently described adjusted cephalic index $(\mathrm{aCl})$, which accounts for altered euryon location. This study tracks the time course of these measures following endoscopic repair with orthotic helmet therapy. The authors hypothesize that $\mathrm{Cl}$ and $\mathrm{aCl}$ show significant regression following endoscope-assisted repair.

METHODS CT scans or 3D photographs of patients with nonsyndromic sagittal synostosis treated before 6 months of age by endoscope-assisted strip craniectomy and postoperative helmet therapy $(n=41)$ were reviewed retrospectively at three time points (preoperatively, 0-2 months after helmeting, and > 24 months postoperatively). The $\mathrm{Cl}$ and aCl were measured at each time point.

RESULTS Mean $\mathrm{Cl}$ and aCl increased from 71.8 to 78.2 and 62.7 to 72.4 , respectively, during helmet treatment $(\mathrm{p}<$ 0.001). At final follow-up, mean $\mathrm{Cl}$ and $\mathrm{aCl}$ had regressed significantly from 78.2 to 76.5 and 72.4 to 69.7 , respectively ( $\mathrm{p}$ $<0.001)$. The $\mathrm{Cl}$ regressed in 33 of 41 cases (80\%) and aCl in 39 of 41 cases (95\%). The authors observed a mean loss of $31 \%$ of improvement in $\mathrm{aCl}$ achieved through treatment. A strong, positive correlation existed between $\mathrm{Cl}$ and $\mathrm{aCl}(\mathrm{R}$ $=0.88$ ).

CONCLUSIONS Regression following endoscope-assisted strip craniectomy with postoperative helmet therapy commonly occurs in patients with sagittal synostosis. Future studies are required to determine whether duration of helmet therapy or modifications in helmet design affect regression.
\end{abstract}

https://thejns.org/doi/abs/10.3171/2018.7.PEDS18195

KEYWORDS sagittal synostosis; cephalic index; plastic surgery; endoscopic; regression; craniofacial

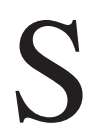
AgitTal synostosis, a condition characterized by premature fusion of the sagittal suture, is the most prevalent form of craniosynostosis, occurring in approximately 1 of every 5000 live births., ${ }^{729}$ In normal development, anatomical fusion of the sagittal suture typically does not occur until the end of puberty. In the setting of this pathological fusion, however, rapid brain development within the first year of life directs cranial growth in the anteroposterior dimension, resulting in a characteristic scaphocephalic head appearance. Features of this deformity include frontal bossing, temporal protrusion, coronal constriction, and occipital protuberance. ${ }^{26}$ Over the last 20 years, surgical correction has evolved from a set of exclusively open procedures to now include minimally invasive spring- and endoscope-assisted procedures, which have demonstrated equivalent short-term outcomes. ${ }^{11,15}$

The conventionally reported measure of both scaphocephalic severity and efficacy of surgical intervention is cephalic index (CI), calculated as the ratio of biparietal to occipitofrontal diameters with a normal range of 75 to 85. .,, $14^{14}$ However, recent studies have demonstrated the insufficiency of CI as an outcome measure, largely because of its inability to account for several cranial abnormalities associated with sagittal synostosis. ${ }^{5,6}$ The adjusted CI $(\mathrm{aCI})$ is a recently described metric that more accurately assesses preoperative severity and postoperative outcomes by accounting for ideal euryon location. ${ }^{5}$

While mild regression of CI has been documented fol- 
lowing open repair, ${ }^{1,7}$ assessment of long-term outcomes following endoscopic repair has not been published. This study aims to determine whether traditional and adjusted CI values of patients managed with endoscope-assisted strip craniectomy and postoperative helmet therapy show regression following completion of treatment. A secondary aim is to characterize the time course of aCI in the years following treatment.

\section{Methods}

\section{Patient and Study Criteria}

IRB approval was obtained prior to the commencement of this study. Patients with sagittal synostosis who had undergone endoscope-assisted strip craniectomy and postoperative helmet therapy were identified from the Craniofacial Anomalies Archive at St. Louis Children's Hospital. Patients treated between October 2006 and August 2014 were considered. Patients were required to meet the following criteria: 1) age under 6 months at the time of operation, 2) preoperative imaging, 3) imaging within 2 months of postoperative helmet therapy completion (initial postoperative), and 4) imaging beyond 24 months postoperatively (final postoperative). Imaging for individual patients was restricted to a single modality: CT or 3D photography (3D photo), at all three time points. Patients whose imaging contained motion artifact or obstruction of the glabella, opisthocranion, or porion were excluded. Forty-one eligible patients with nonsyndromic sagittal synostosis treated by endoscope-assisted strip craniectomy ${ }^{28}$ and postoperative orthotic helmet therapy met the inclusion criteria. Of this group, CT scans were analyzed for 8 patients and 3D photographs for 33 patients.

\section{Data Collection}

CT data were three-dimensionally reconstructed and analyzed using Analyze software (version 12.0, Mayo Clinic). Three-dimensional photographs were obtained using a 4-pod 3dMD camera system and analyzed using Vultus software (3dMD). All reconstructions were oriented to the Frankfort horizontal plane. Point measure tools were utilized to determine the 3D location of the required landmarks: glabella, opisthocranion, vertex, traditional euryons, and ideal euryon locations. ${ }^{5}$

Four measurements were calculated: 1) biparietal width, the distance between euryons; 2) the distance between ideal euryon locations; ${ }^{5} 3$ ) anteroposterior length, the distance from glabella to opisthocranion; and 4) cranial height, the vertical distance from nasion to vertex. Ideal euryon location was set as previously described by Dvoracek et al., defined by the average vertical point of maximum width and horizontal point of maximum width in controls. ${ }^{5}$ The CI was calculated by dividing biparietal width by anteroposterior length and multiplying by 100 . The aCI was calculated in the same manner as the traditional CI, except for the use of ideal euryon location in biparietal diameter measurement (Fig. 1). Measurements were made at preoperative, initial postoperative, and final postoperative time points in all patients.

Repeat measurements of 10 CT data sets and 4 3D pho-
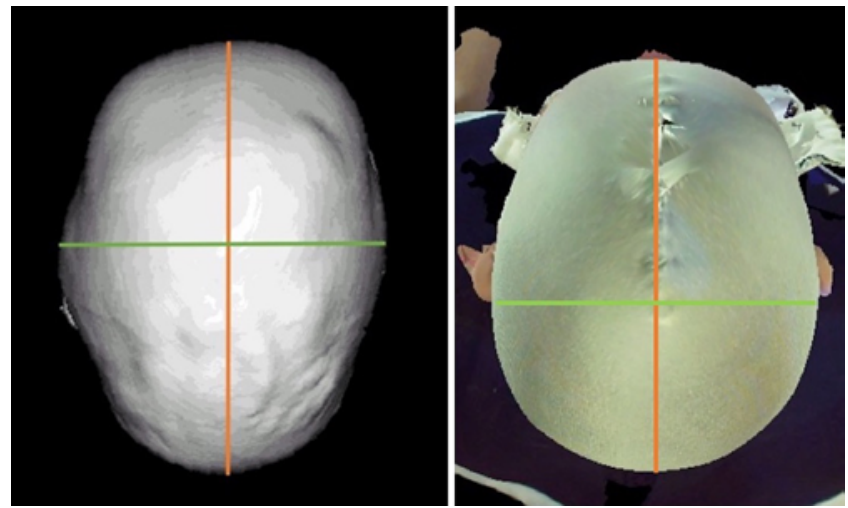

FIG. 1. Vertex view of a scaphocephalic patient CT scan (left) and 3D photograph (right). Anteroposterior length (orange line) and traditional biparietal width (green line) are shown. Figure is available in color online only.

tos were performed to gauge intrarater reliability. These measurements were spaced 1 week apart in an attempt to blind the rater to previous measurements. Similarly, 10 subject- and date-matched CT and 3D photo pairs were assessed for bias between the scanning modalities. One rater (N.A.P.) performed all measurements.

In the second arm of the study, changes in postoperative aCI over a longer interval were measured to delineate trends during and after treatment. Of the original included patients, a subset of 8 individuals were identified who met the following additional criteria: 1) a minimum of 5 postoperative images, and 2) final follow-up imaging beyond 48 months postoperatively. These additional images were processed in the manner described above. The aCI was calculated at each time point.

\section{Statistical Analysis}

Statistical analysis focused on image time points in addition to traditional and adjusted CIs. Differences between preoperative, initial postoperative, and final postoperative results were evaluated using 1-way repeated-measures ANOVA with Greenhouse-Geisser correction. Differences in proportions were obtained using Fisher's exact test. The Holm-Bonferroni correction method was applied to control the family-wise error rate. Significance was set a priori at $\alpha=0.05$. Statistical analysis was performed using SPSS (version 22, IBM Corp.).

\section{Helmet Protocol}

A custom-made molding helmet is used to guide calvarial growth as the brain expands during development, allowing for transformation of a scaphocephalic appearance to a normal head shape. By providing direct contact to the frontal and occipital midline regions of the cranium, the helmet promotes the biparietal expansion necessary to achieve a superior aesthetic outcome. Helmet therapy is typically initiated within 1-3 days postoperatively. We recommend that the helmet be worn for at least 23 hours per day until the age of 1 , when the majority of brain growth has occurred. ${ }^{27}$ During this period, 2-4 orthotic helmets are typically required to account for 
Pickersgill et al.

TABLE 1. Cls and age at studied time points

\begin{tabular}{lccc}
\hline Time Point & $\mathrm{Cl}$ & $\mathrm{aCl}$ & Age (mos) \\
\hline Preop & $71.8 \pm 4.1$ & $62.7 \pm 3.6$ & $2.3 \pm 1.5$ \\
\hline Initial postop & $78.2 \pm 4.8$ & $72.4 \pm 4.4$ & $15.3 \pm 4.2$ \\
\hline Final postop & $76.5 \pm 4.6$ & $69.7 \pm 4.3$ & $47.3 \pm 13.9$ \\
\hline
\end{tabular}

Values are expressed as mean $\pm \mathrm{SE}$ for all parameters. Mean traditional and adjusted $\mathrm{Cl}$ values were significantly different between time points $(p<0.001)$.

growth and changes during cranial expansion. ${ }^{23}$ In general, our orthotists report that patients with operative ages of 2-3 months require 3-4 helmets, while older patients aged $>4$ months require $2-3$ helmets.

\section{Results}

\section{Patient Demographics and Follow-Up Times}

In the first arm of the study, traditional and adjusted CIs were measured at the three designated time points. The sample group consisted of 31 boys and 10 girls. Eight patients (19.5\%) underwent narrow vertex suturectomy and 33 patients $(80.5 \%)$ underwent wide vertex suturectomy. Mean age ( \pm standard deviation) at the time of surgery was $3.2 \pm 1.0$ months in the wide vertex group and $4.1 \pm$ 1.3 months in the narrow vertex group. Mean ( \pm standard deviation) postoperative follow-up duration was 11.9 \pm 4.1 months for initial imaging and $43.9 \pm 13.8$ months for final imaging. Helmet therapy lasted an average of 7.3 \pm 2.5 months. Forty of 41 patients were reported as compliant with the helmet protocol. Patient age at each time point is listed in Table 1. For reference, a sample series of
$3 \mathrm{D}$ photographs at the designated time points is presented in Fig. 2.

In the second arm of the study, both indices were calculated at additional time points over an extended postoperative period. This subgroup of patients consisted of 7 boys and 1 girl. The duration of postoperative monitoring in this group was $63.1 \pm 15.5$ months. The mean number of time points was $6.5 \pm 1.2$.

Reliability was excellent for both measures and both modalities. With CT, the 2-way random intraclass correlation coefficients (ICCs) were $0.975(\mathrm{CI})$ and $0.976(\mathrm{aCI})$. With 3D photos, the ICCs were $0.996(\mathrm{CI})$ and $0.956(\mathrm{aCI})$. Comparisons between modalities showed slightly higher values for both CI $(0.6 \pm 1.3)$ and aCI $(2.0 \pm 1.1)$ in CT compared with 3D photos.

\section{Traditional and Adjusted CI Regression}

A strong positive correlation existed between traditional and adjusted $\mathrm{CI}(\mathrm{R}=0.88, \mathrm{p}<0.001)$. Post hoc testing showed that upon completion of postoperative helmet therapy, traditional and adjusted CI had improved by 6.4 and 9.7, respectively $(\mathrm{p}<0.001)$. At longer-term follow-up, traditional and adjusted CI had regressed by 1.7 and 2.7, respectively ( $p<0.001$; Fig. 3). Results of the first arm of the study are summarized in Table 1. Post hoc testing confirmed that aCI was significantly less than the traditional $\mathrm{CI}$ at each time point $(\mathrm{p}<0.001)$.

There were no statistically significant differences in the degree of regression following helmeting between the wide and narrow vertex suturectomy groups. CI regression was 1.5 in the wide group and 2.6 in the narrow group $(\mathrm{p}=0.253)$, while aCI regression was 2.7 in the wide group and 3.0 in the narrow group $(\mathrm{p}=0.721)$. Additionally, the
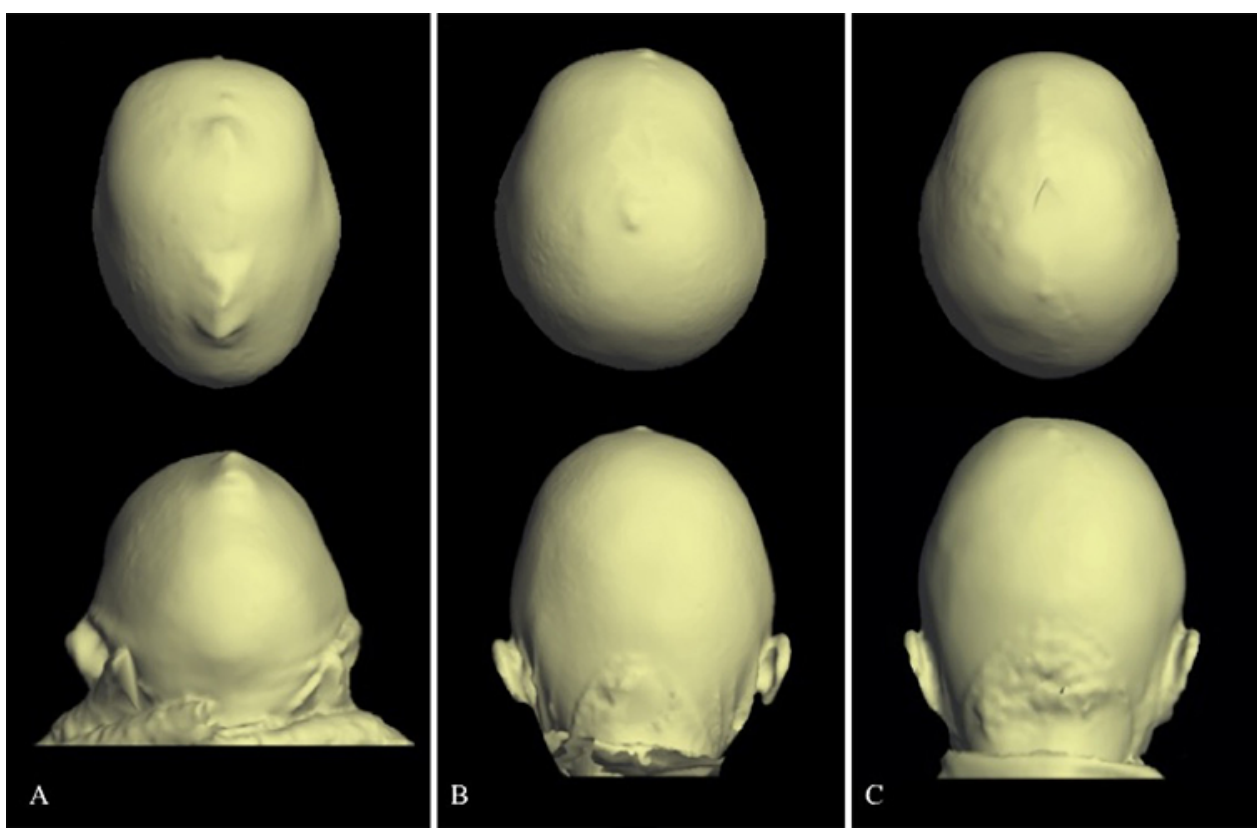

FIG. 2. Series of vertex and posterior views of 3D photographs of a patient with sagittal synostosis taken at specified time points: preoperative $(\mathbf{A})$, initial postoperative $(\mathbf{B})$, and final postoperative $(\mathbf{C})$. Lumps are due to imperfect skullcap fit. Figure is available in color online only. 


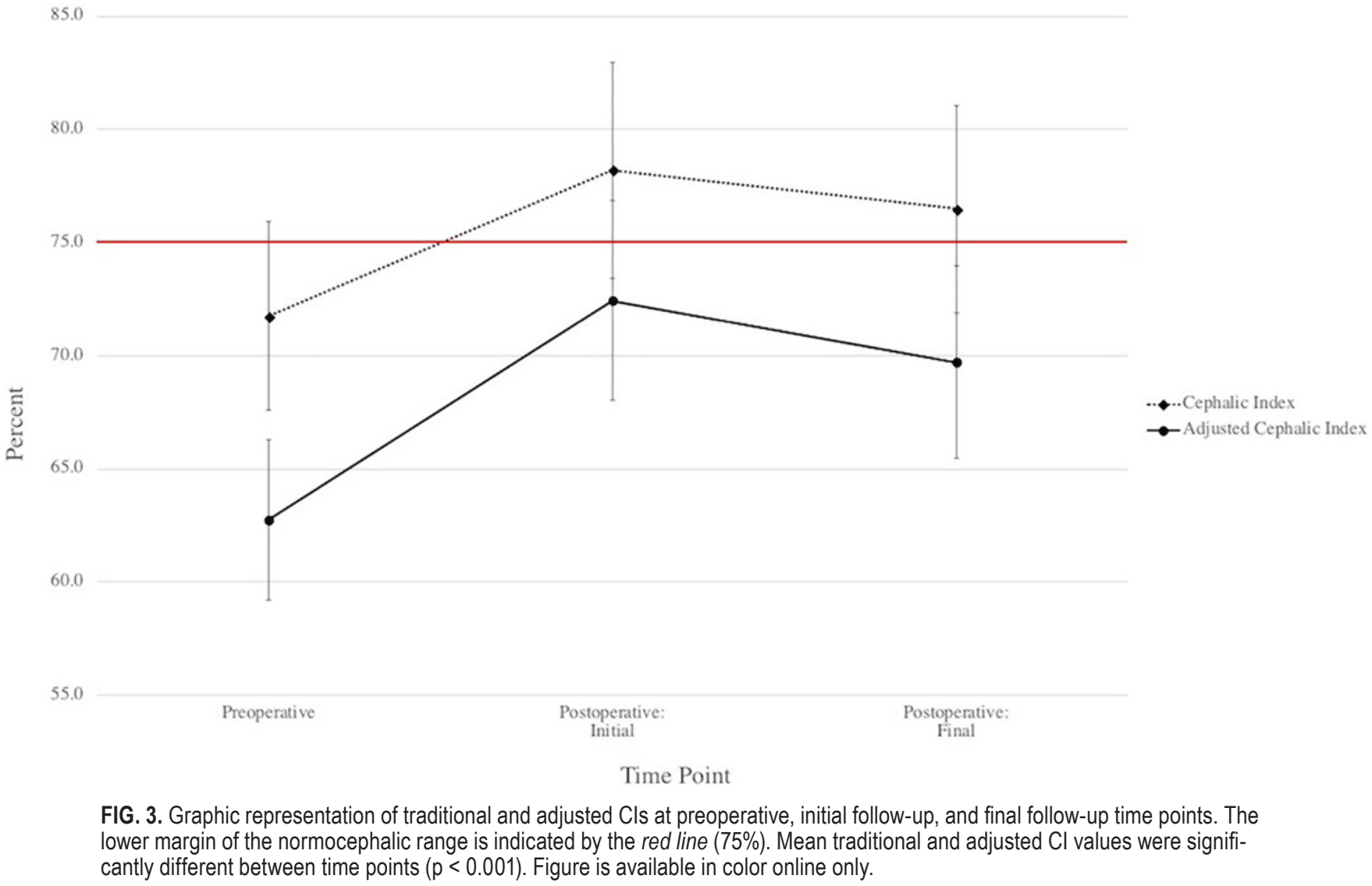

proportions of patients with final $\mathrm{CI}$ and aCI within the normal range were not significantly different between the two repair types $(p=0.115)$.

CI regressed in 33 cases (80\%) and aCI in 39 cases (95\%) between completion of postoperative helmet therapy and final postoperative follow-up. At the latest followup evaluation, both traditional and adjusted CIs were below the normal range in 22 cases (54\%). Despite prevalent regression, at final follow-up both indices remained higher than preoperative values in all patients $(\mathrm{p}<0.001)$. The mean overall postoperative improvements in traditional and adjusted CIs at the latest follow-up were 4.7 and 7.0, respectively.

In the second arm of the study, postoperative aCI was measured in a subgroup $(n=8)$ of patients at shorter intervals for a minimum of 48 months (Fig. 4). Two-way repeated-measures ANOVA with Bonferroni correction showed no significant difference in traditional or adjusted CI between this subgroup and the entire study group.

Mean time to final follow-up was $63.1 \pm 15.5$ months. Mean maximum improvement in aCI occurred $5.3 \pm 2.3$ months following surgery with a mean increase of $13.8 \pm$ 4.4. Mean aCI had regressed by 1.9 at 10 months and 4.0 at 48 months postoperatively, indicating continued decline into the extended postoperative period. At final follow-up, mean aCI had regressed by 4.3 , signifying a loss of $31 \%$ of quantitative improvement achieved through surgical intervention and orthotic therapy. Age at completion of helmet therapy was poorly correlated with $\mathrm{CI}(\mathrm{R}=0.12, \mathrm{p}=0.47)$ and $\mathrm{aCI}(\mathrm{R}=0.02, \mathrm{p}=0.89)$. The results of this arm of the study are summarized in Table 2 .

\section{Discussion}

The cranial deformity associated with sagittal synostosis can have a lasting psychosocial impact on developing children. ${ }^{17}$ Surgical intervention is aimed at minimizing these effects through restoration of normal skull shape and appearance as well as prevention or reduction of increased intracranial pressure. ${ }^{19}$ Permanent, successful correction of scaphocephaly is therefore paramount to the healthy development of patients affected by this condition.

\section{Traditional and Adjusted CI Regression}

Several studies have assessed long-term postsurgical outcomes based on the traditional CI,,$^{1,2,15,26}$ but none have used aCI, which accounts for altered euryon location. Furthermore, these studies have focused solely on outcomes following open surgical procedures. Over the past 20 years, however, there has been a sharp rise in the use of minimally invasive endoscopic techniques to treat craniosynostosis due to shorter surgical times, minimal blood loss, and smaller incisions. , $^{3,20}$ Given the growing role of endoscopic procedures in the treatment of sagittal synostosis, an objective of this study was to conduct an equal and adequate investigation of its postoperative anthropometric outcomes.

Following completion of orthotic helmet therapy, in- 


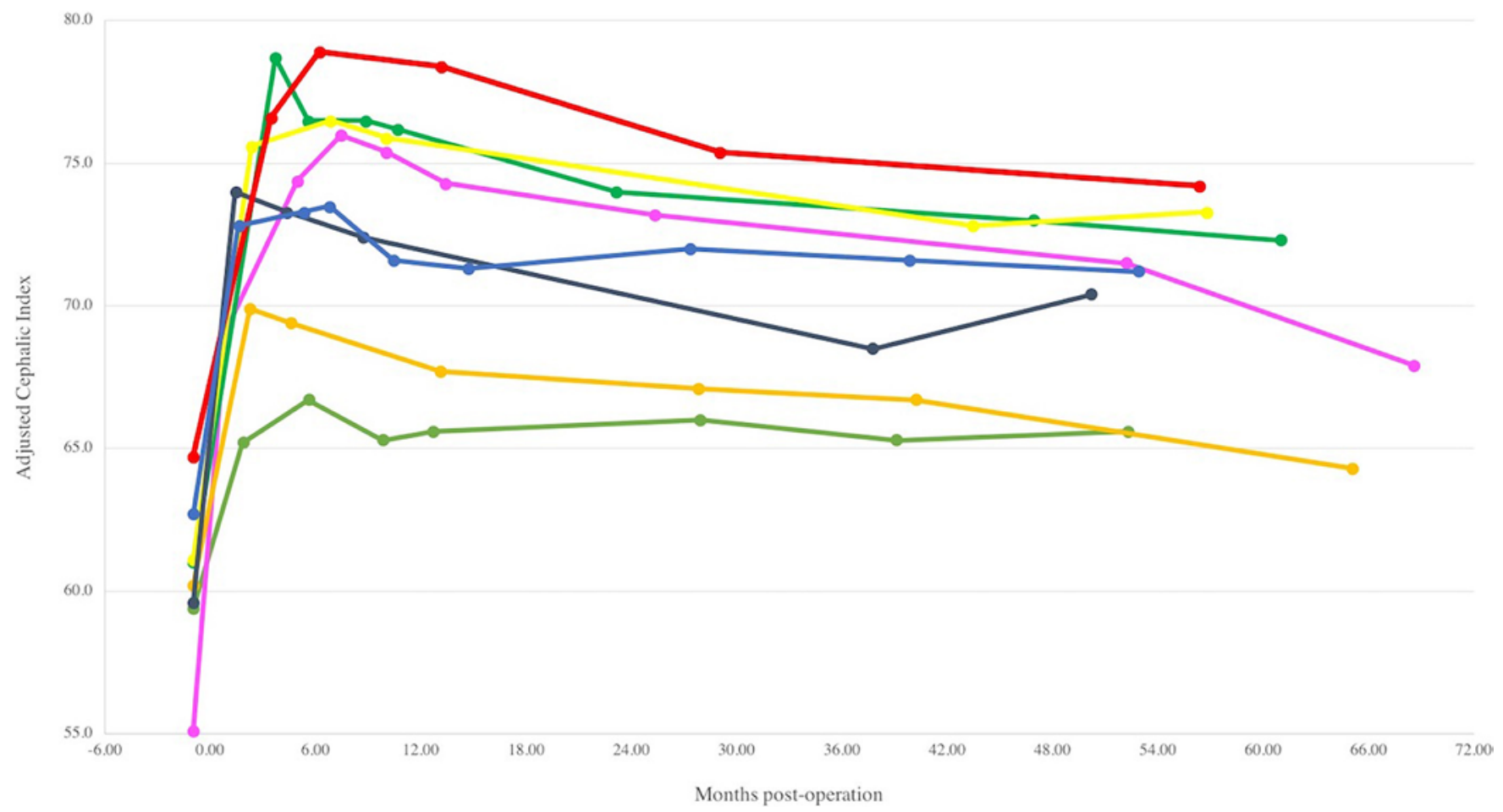

FIG. 4. Graphic representation of postoperative changes in $\mathrm{aCl}$ over time at shorter intervals in 8 patients. Figure is available in color online only.

creases in both traditional and adjusted CI were observed. As with open procedures, ${ }^{1,10,18}$ peak improvement occurred in the early postoperative period.

Following initial improvement, however, both measures began to decline. Interestingly, aCI declined more than the traditional CI (2.7\% vs $1.7 \%)$, suggesting an underestimation of regression by the latter metric. A similar degree of traditional CI regression (1.8\%) following open repair (vertex craniectomy and barrel-stave osteotomy) was reported by Agrawal et al. ${ }^{1}$ Interestingly, even less regression $(0.46 \%)$ was reported by Fearon et al. following single-stage posterior cranial vault remodeling. ${ }^{7}$ Ridgway et al. also reported a decline in CI of $1 \%$ approximately 3 years following endoscopic suturectomy. ${ }^{25}$ As shown in Fig. 3, mean aCI at final follow-up was below the reference range despite the traditional CI remaining within normal limits. These results support the notion that the traditional $\mathrm{CI}$ is an insufficient measure of overall appearance, likely because of failure to account for biparietal narrowing. Multiple studies report little to no correlation between CI and subjective assessments of severity. ${ }^{5,6,26}$ One explanation is that family members do not note the parietal deformity after hair growth but are more concerned with frontal bossing. With the endoscopic procedure, biparietal expansion guided by helmet therapy normalizes frontal morphology over a 1- to 2-year period..$^{15}$ This is similar to open techniques that avoid a frontal craniotomy as described by Khechoyan et al. ${ }^{12}$

Our analysis found similar regression of both traditional and adjusted CIs in patients who underwent narrow vertex suturectomy compared to wide vertex suturectomy.
This finding is consistent with a previous study by this group that found no difference in postoperative CI between the two procedures at 1-year follow-up. ${ }^{4}$

\section{Time-Course of aCl}

The second arm of this study demonstrates that maximal improvement occurs within the first 6 months of surgery, after which the aCI begins to decline steadily (Fig. 4). This is in concordance with previous studies of the CI..$^{14,26,28}$ Abnormal skull growth is common following surgical repair of sagittal synostosis..$^{24,26}$ Given these findings, it is possible that modification of current orthotic therapy regimens may lead to improved cephalometric outcomes.

Although surgeons aim to achieve a CI of 75 and above with treatment, our results indicate that many patients regress beyond this threshold, and even more so when $\mathrm{aCI}$ is considered. In $95 \%$ of patients, the initial increase in aCI through endoscope-assisted strip craniectomy and postoperative helmet therapy was followed by a period of regres-

TABLE 2. Adjusted Cl in long-term study subgroup

\begin{tabular}{|c|c|c|c|}
\hline Preop aCl & Max aCl & $\begin{array}{c}\mathrm{aCl} \text { at } 10 \mathrm{Mos} \\
\text { Postop* }\end{array}$ & $\begin{array}{c}\text { aCl at } 48 \text { Mos } \\
\text { Postop } †\end{array}$ \\
\hline $60.5 \pm 2.8$ & $74.3 \pm 4.2$ & $72.4 \pm 4.7$ & $70.3 \pm 3.6$ \\
\hline
\end{tabular}


sion, resulting in the loss of approximately one-third of the quantitative progress. Studies of open procedure outcomes also suggest that overcorrection of the deformity may be beneficial in the maintenance of a normocephalic appearance in the years following treatment. ${ }^{26,28}$

\section{Limitations}

There were several limitations of this study. First was our inability to control for possible normal developmental CI decline, which has been shown in observational studies of healthy children, albeit to a lesser degree. ${ }^{13,16}$ Second, intrinsic flaws in imaging include motion artifact and 3D photograph distortion due to hair interference (which is only partially resolved with the use of a tight-fitting stockinet cap) and motion artifact. A substantial number of patients with adequately timed imaging studies were excluded from the study for this reason. Finally, two different imaging modalities were used. This limitation was mitigated by an inclusion criterion restricting each participant to a single modality. Additionally, previous research has shown no bias between CIs measured by these two imaging techniques. ${ }^{22}$

\section{Future Directions}

Following completion of this study, the authors made changes in helmet design to concentrate on increasing CI at ideal euryon location. Additionally, our orthotists now measure both traditional and adjusted CI at each follow-up visit. Further investigation is required to assess whether changes to the helmet protocol (e.g., duration of helmet therapy and helmet design) affect patient outcomes such as cosmetic appearance, traditional and adjusted CI, and other morphological metrics.

\section{Conclusions}

Regression toward scaphocephaly following endoscope-assisted strip craniectomy and postoperative helmet therapy commonly occurs in patients with sagittal synostosis. The implementation of the aCI revealed more significant regression than previously observed with the traditional CI. Further studies are required to determine whether duration of helmet therapy and modifications in helmet design affect regression. Results of continued investigation could impact future recommended protocols for postoperative management of sagittal synostosis.

\section{Acknowledgments}

The first author was funded by the Dean's Fellowship grant of Washington University in St. Louis. Research reported in this publication was also supported by the Washington University Institute of Clinical and Translational Sciences grant UL1 TR000448 from the National Center for Advancing Translational Sciences of the National Institutes of Health and Children's Surgical Sciences Institute.

\section{References}

1. Agrawal D, Steinbok P, Cochrane DD: Long-term anthropometric outcomes following surgery for isolated sagittal craniosynostosis. J Neurosurg 105 (5 Suppl):357-360, 2006

2. Albright AL, Towbin RB, Shultz BL: Long-term outcome after sagittal synostosis operations. Pediatr Neurosurg 25:78-82, 1996

3. Di Rocco C, Tamburrini G, Pietrini D: Blood sparing in craniosynostosis surgery. Semin Pediatr Neurol 11:278-287, 2004

4. Dlouhy BJ, Nguyen DC, Patel KB, Hoben GM, Skolnick GB, Naidoo SD, et al: Endoscope-assisted management of sagittal synostosis: wide vertex suturectomy and barrel stave osteotomies versus narrow vertex suturectomy. J Neurosurg Pediatr 25:674-678, 2016

5. Dvoracek LA, Skolnick GB, Nguyen DC, Naidoo SD, Smyth $\mathrm{MD}$, Woo AS, et al: Comparison of traditional versus normative cephalic index in patients with sagittal synostosis: measure of scaphocephaly and postoperative outcome. Plast Reconstr Surg 136:541-548, 2015

6. Fearon JA, Ditthakasem K, Herbert M, Kolar J: An appraisal of the cephalic index in sagittal craniosynostosis, and the unseen third dimension. Plast Reconstr Surg 140:138-145, 2017

7. Fearon JA, McLaughlin EB, Kolar JC: Sagittal craniosynostosis: surgical outcomes and long-term growth. Plast Reconstr Surg 117:532-541, 2006

8. Gray DL, Songster GS, Parvin CA, Crane JP: Cephalic index: a gestational age-dependent biometric parameter. Obstet Gynecol 74:600-603, 1989

9. Haas LL: Roentgenological skull measurements and their diagnostic applications. Am J Roentgenol Radium Ther Nucl Med 67:197-209, 1952

10. Kaiser G: Sagittal synostosis - its clinical significance and the results of three different methods of craniectomy. Childs Nerv Syst 4:223-230, 1988

11. Kaufman BA, Muszynski CA, Matthews A, Etter N: The circle of sagittal synostosis surgery. Semin Pediatr Neurol 11:243-248, 2004

12. Khechoyan D, Schook C, Birgfeld CB, Khosla RK, Saltzman $\mathrm{B}$, Teng CC, et al: Changes in frontal morphology after single-stage open posterior-middle vault expansion for sagittal craniosynostosis. Plast Reconstr Surg 129:504-516, 2012

13. Koizumi T, Komuro Y, Hashizume K, Yanai A: Cephalic index of Japanese children with normal brain development. J Craniofac Surg 21:1434-1437, 2010

14. Kurmanavicius J, Wright EM, Royston P, Wisser J, Huch R, Huch A, et al: Fetal ultrasound biometry: 1. Head reference values. Br J Obstet Gynaecol 106:126-135, 1999

15. Le MB, Patel K, Skolnick G, Naidoo S, Smyth M, Kane A, et al: Assessing long-term outcomes of open and endoscopic sagittal synostosis reconstruction using three-dimensional photography. J Craniofac Surg 25:573-576, 2014

16. Likus W, Bajor G, Gruszczyńska K, Baron J, Markowski J, Machnikowska-Sokołowska M, et al: Cephalic index in the first three years of life: study of children with normal brain development based on computed tomography. Sci World J 2014:502836, 2014

17. Magge SN, Westerveld M, Pruzinsky T, Persing JA: Longterm neuropsychological effects of sagittal craniosynostosis on child development. J Craniofac Surg 13:99-104, 2002

18. Marsh JL, Jenny A, Galic M, Picker S, Vannier MW: Surgical management of sagittal synostosis. A quantitative evaluation of two techniques. Neurosurg Clin N Am 2:629-640, 1991

19. Massimi L, Tamburrini G, Caldarelli M, Di Rocco C: Effectiveness of a limited invasive scalp approach in the correction of sagittal craniosynostosis. Childs Nerv Syst 23:1389-1401, 2007

20. Meier PM, Goobie SM, DiNardo JA, Proctor MR, Zurakowski D, Soriano SG: Endoscopic strip craniectomy in early infancy: the initial five years of anesthesia experience. Anesth Analg 112:407-414, 2011

21. Meier PM, Guzman R, Erb TO: Endoscopic pediatric neu- 
rosurgery: implications for anesthesia. Paediatr Anaesth 24:668-677, 2014

22. Mendonca DA, Naidoo SD, Skolnick G, Skladman R, Woo AS: Comparative study of cranial anthropometric measurement by traditional calipers to computed tomography and three-dimensional photogrammetry. J Craniofac Surg 24:1106-1110, 2013

23. Nguyen DC, Farber SJ, Skolnick GB, Naidoo SD, Smyth MD, Kane AA, et al: One hundred consecutive endoscopic repairs of sagittal craniosynostosis: an evolution in care. J Neurosurg Pediatr 20:410-418, 2017

24. Panchal J, Marsh JL, Park TS, Kaufman B, Pilgram T, Huang SH: Sagittal craniosynostosis outcome assessment for two methods and timings of intervention. Plast Reconstr Surg 103:1574-1584, 1999

25. Ridgway EB, Berry-Candelario J, Grondin RT, Rogers GF, Proctor MR: The management of sagittal synostosis using endoscopic suturectomy and postoperative helmet therapy. J Neurosurg Pediatr 7:620-626, 2011

26. Schmelzer RE, Perlyn CA, Kane AA, Pilgram TK, Govier D, Marsh JL: Identifying reproducible patterns of calvarial dysmorphology in nonsyndromic sagittal craniosynostosis may affect operative intervention and outcomes assessment. Plast Reconstr Surg 119:1546-1552, 2007

27. Sgouros S, Hockley AD, Goldin JH, Wake MJ, Natarajan K: Intracranial volume change in craniosynostosis. J Neurosurg 91:617-625, 1999

28. Shah MN, Kane AA, Petersen JD, Woo AS, Naidoo SD, Smyth MD: Endoscopically assisted versus open repair of sagittal craniosynostosis: the St. Louis Children's Hospital experience. J Neurosurg Pediatr 8:165-170, 2011

29. Wilkie AO, Wall SA: Craniosynostosis: novel insights into pathogenesis and treatment. Curr Opin Neurol 9:146-152, 1996

\section{Disclosures}

Dr. Patel is a consultant for Stryker CMF and a paid speaker for Hanger Clinic. Dr. Naidoo is a paid speaker for Hanger Clinic and Orthomerica.

\section{Author Contributions}

Conception and design: Patel, Skolnick, Naidoo. Acquisition of data: Patel, Pickersgill, Skolnick, Smyth. Analysis and interpretation of data: Pickersgill, Skolnick. Drafting the article: Pickersgill. Critically revising the article: all authors. Reviewed submitted version of manuscript: all authors. Approved the final version of the manuscript on behalf of all authors: Patel. Statistical analysis: Pickersgill, Skolnick. Administrative/technical/material support: Skolnick. Study supervision: Patel, Skolnick.

\section{Supplemental Information \\ Previous Presentations}

Parts of this study were previously presented at the International Society of Craniofacial Surgeons meeting in Cancun, Mexico, in 2017 and at the American Cleft Palate-Craniofacial Association's 76th Annual Meeting in Pittsburgh, Pennsylvania, in 2018 (both in abstract form).

\section{Correspondence}

Kamlesh B. Patel: Washington University in St. Louis, MO. kamlesh.patel@wustl.edu. 